\title{
Spatial Memory of Taps on the Fingers
}

\author{
P. Venkata Prakash N*, U.G. Scholar, Manoj Kumar D.S.** \\ *Department of Computer Science and Engineering \\ **Saveetha School of Engineering, Saveetha University
}

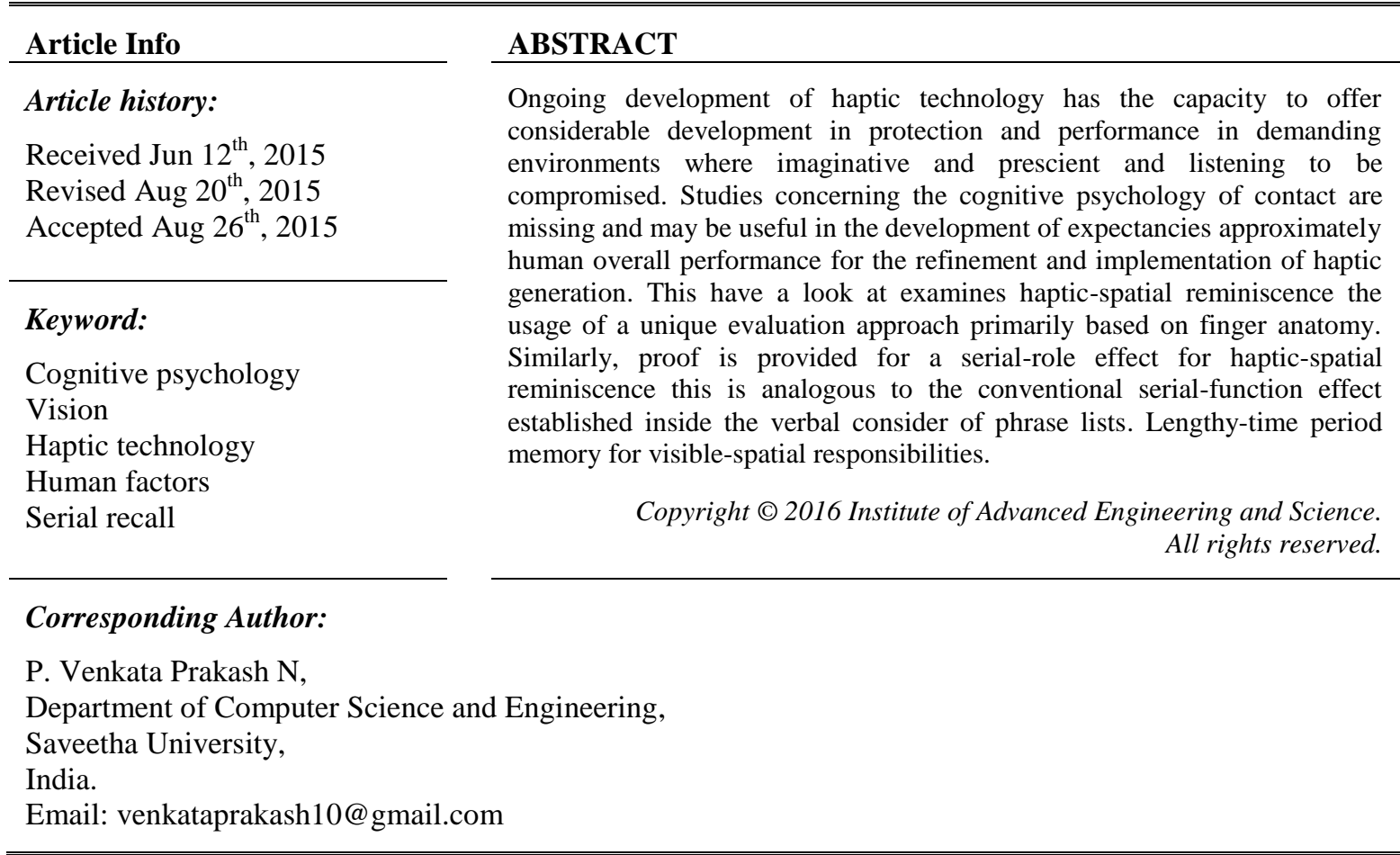

\section{INTRODUCTION}

PERCEPTUAL statistics elicited by way of energetic touch, i.e., haptics, is vital in lots of every day sports and is contemplated in a disproportionately large vicinity of the primary somatosensory cortex dedicated to the arms as compared to different outside surfaces of the body. however, there is commonly little data inside the literature of cognitive psychology for touch compared to vision and hearing, that are represented via large studies on interest, pattern popularity, memory, knowledge representation, language, and hassle solving a state of affairs that has changed little since the seminal try of to estimate limits of information processing as a function of memory capacity. Despite the fact that haptics is a complex activity, our fingers are facile in conveying perceptual facts approximately the environ- ment.

Haptics accommodates numerous fundamental exploratory tactics, consisting of application of lateral movement, pres- certain, and contour following to shape the precepts of texture, hardness, and form, respectively. Sensory comments, motor movement, and cognitive associations are incorporated to form a haptic percept; as an example, with handiest minimum attempt, an in keeping with- son can use haptics to reliably differentiate among a base- ball and a billiard ball. Given its anatomical layout, the hand is ideal for the experimental evaluation of cognitive methods for contact. Wonderful regions of the hand are clearly marked, e.g., each finger incorporates 3 phalanges creating a herbal $3 \hat{\text { A }} 4$ matrix of rows and columns. whilst others have used the fingers to investigate variables influencing localization of tac- tile stimuli or finger tapping for neuroimaging studies of motor activity the layout used in this have a look at is a novel technique to collect simple descriptive facts approximately haptic-spatial memory. In comparison to passive touch, haptics is the active technique- ing of records about the environment the use of cutaneous and kinesthetic inputs derived from purposive behaviour this might include proprioception, e.g., voluntary motor pastime involved in probing the surface of an object with the arms. However, a character actively taking note of styles of tactile stimulation applied to the floor of the body is engaged in haptic conduct through ultimate vigilant 
for the reception of tactile facts. as an instance, an alternative communique device (ACS) referred to as Taptalk changed into evolved the usage of the phalangeal-finger matrix described above wherein individuals communicate reciprocally by attending to and administering styles of faucets at the fin- gers , the "speaker" typically uses seasoned-prioception to administer faucets comprising particular spatial styles at the same time as the "listener" actively attends to the cutane- ous spatial impressions created via the patterns of tapping.

Taptalk is based on haptic-spatial reminiscence and is comfortably adaptable to technological improvements requiring vigilant attention to tactile alerts. The software of haptic era stages from computer display to an expansion of labor environments. For example, Tactons, or tactile icons, seek advice from nonvisual, tactile message presentations that offer an additional modality in a pc consumer interface complementing photo and auditory output. Haptic technology has been developed and utilized by others to talk environmental variables to people in critical environments that gift heavy sensory load for the visual and auditory modalities. An example of such an application is the Tactile Situation recognition gadget (TSAS) whereby a combat pilot carrying tactile stimulators about the torso can process information regarding frame function, altitude, speed, or even target location; the TSAS gives the pilot an extra source of data thru haptics that poten- tially improves protection and overall performance.

The feel of contact can complement or make amends for input from different sensory modalities. For example, touch and imaginative and prescient paintings collectively to facilitate professional motor interest, which include sewing or carving. similarly, haptics can com- pensate in part for capabilities typically related to the visual or auditory modality; for instance, someone who's blind can use the Braille machine to get right of entry to the written phrase at the same time as someone who's deaf can use vibrotactile stimulation to appreciate the sector of track.In truth, record that dependable direction integration can be performed from tactile cues by myself by using integrating motion over the years, so that indi-viduals ought to infer multiple geometric characteristics of a simulated triangle. Spatial facts derived by way of different sensorymodalities can be encoded in corresponding reference frames that interact with every different in working memory to shape a coherent percept, e.g., a percept that effects whilst examining a statue by using touch and imaginative and prescient. however, the nature of this interaction is not apparent; the usage of a cross-modal visuo- haptic spatial rotation undertaking, located that seen or touched gadgets do no longer want to be bodily aligned to obtain opti- mal object-identity overall performance, yet the visual and haptic reference frames though directly influence each other.

Underscoring the importance of reciprocal go- modal interactions between touch and vision, movement after- consequences brought on in the contact modality can affect the according to- ception of analogy movement aftereffects inside the visible mode, a phenomenon that also works reciprocally. Fundamental research on the cognitive techniques for touch (together with move-modal interactions) can beautify the ongo- ing development of haptic technology. The method of assessment for haptic-spatial memory in this study empha- sizes ecological validity associated with the practical application of haptics, e.g., two individuals communicating with Taptalk. While a tap of a phalange results in a three- dimensional depression on the surface of the finger, multiple taps within a small temporal interval (e.g., 4 seconds) across different phalanges produce a haptic-spatial pattern in an approximate two-dimensional plane. Immediate recall of a particular haptic-spatial pattern constitutes an explicit (short-term) memory that depends on the relative contribu- tion of each tap that can be coded with respect to finger (pinky, ring, middle, or index), hand (left or right), and serial position (first, second, third, or fourth). Thus, this exploratory study of hapticspatial memory by design examines the relative contribution of finger, hand, and serial position. Specifically, three hypotheses are examined in this study of haptic-spatial memory whereby participants actively attend to tactile patterns. The first hypothesis suggests that haptic-spatial memory performance differs depending on stimulus presentation among fingers and between hands.

The speculative basis for a finger difference in part is found in the innervation pattern of the hand the brachial plexus descends the arm and branches into the medial, ulnar, and radial nerves that comprise a complex pattern of receptive fields among the fingers. Furthermore, it is reasonable to speculate that a source of systematic variance in recall performance between hands can be attributed in part to any differences in the separate somatosensory pathways linking tactile sensory input with contralateral cortices [1]; for example, found that for a simple visuo-spatial encoding.

\section{METHODS AND MATERIALS}

\subsection{Participants}

Forty-two females and 14 men participated in our take a look at, with a mean age of M 1/4 20.97 years (SD 1/4 four.04) ranging from 18 to 41 . Individuals were recruited from undergraduate psychology training and received greater credit for their participation. 


\subsection{Handedness}

The prevalence of left-hand dominance in the general population is relatively low, ranging approximately from 5 to 10 percent. With this in mind, a hypothesis predicting differences between left- and right-handed participants were not evaluated in this study. In the present study, seven (n 1/4 7) participants self-reported that they were left-handed and the remaining participants (n 1/4 49) self-reported that they were right-handed. Thus, by design, the results of this study stand without a statistical consideration of handedness; subsequent studies may elect to attain a much larger sample sizes to afford a reasonable representation of the left-hand-dominant population.

\subsection{Materials}

\subsubsection{Patterns}

A sample contains a completely unique order of 4 finger faucets. Each of 4 arms is tapped as soon as and most effective one time in a given pattern; role refers to whether a selected finger is tapped first, 2d, 1/3, or fourth in a sequence for a specific sample; all faucets are administered to the center phalange (whilst administering the patterns, the technician listened to a metronome via a pair of headphones which will maintain a tapping charge of one faucet according to second). A complete of 12 patterns are administered to every hand (each sample is inside hand, i.e., administered to 1 and handiest one hand), counterbalancing function throughout the four hands for every hand, for this reason, every finger is tapped a total of 12 times, three times in each of the 4 feasible positions.

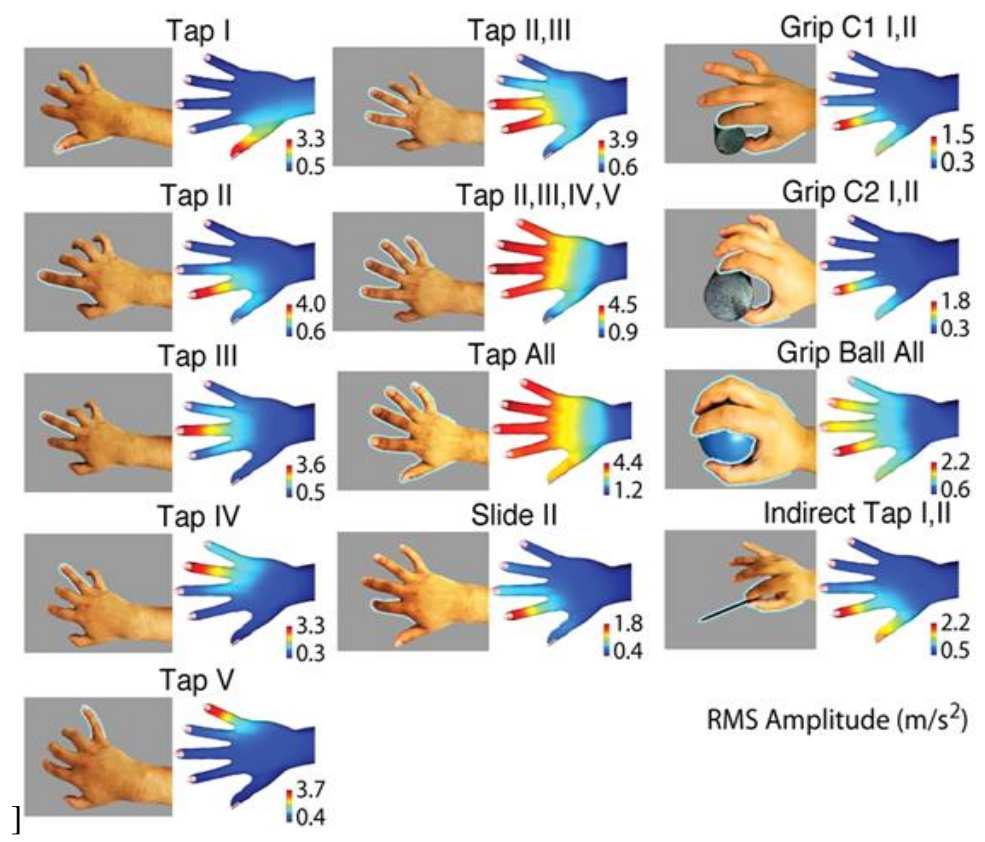

Figure 1. A sample contains a completely unique order of 4 finger faucets

\subsubsection{Colorado Neuropsychology Test (CNT)}

The visible span test (VST) of the Colorado Neuropsychology take a look at changed into used to evaluate participants' express, quick-term visual-spatial memory. Within the VST, 8 squares are displayed (unaligned) on a laptop display screen. Initially, two of the 8 squares flash sequentially; the player attempts to straight away repeat the sample of flashed squares in the best order by clicking on the ideal squares with the mouse, so long as the participant avoids making consecutive mistakes, the range of flashed squares sequentially will increase from to 8 flashing squares; the participant keeps to improve till she makes two consecutive errors. A reminiscence card take a look at (MCT) from the CNT additionally was administered to assess individuals' explicit, long-term visual-spatial reminiscence. just like the kids's card game of attention, this assignment uses twelve pairs of cards ran-domly allotted in a 4-by way of-six matrix, appearing face down on the pc display screen. The object of the sport is to match the pairs of cards. Cards are flipped with a click on of the mouse; handiest two cards may be flipped over at one time. If the cards do not in shape, they mechanically flip face down and the participant attempts again. The participant persists till all pairs of 
playing cards are correctly matched ensuing in a score calculated as the share of correct-pair flips among all tried flips.proper aperture.

\subsection{Procedure}

The procedural order for this observe included the attainment of consent and widespread facts, a finger identity test, the administration of the CNT, and the haptic-spatial memory check. 3 humans have been within the room in the course of each haptic-spatial memory checking out session: a player, a technician, and a videographer. The haptic station contains a desk with a white, three- panel, cardboard display screen hooked up vertically at the tabletop (Figure 1). At the bottom of the display screen had been rectangular apertures, big sufficient for the participant to slide their arms via while blocking the view of the technician. Line drawings of palms, corresponding to the player's left and proper, were affixed at once above the two holes at the participant's aspect of the display. The road drawings were simple outlines of a left or proper hand with black dots focused inside the middle phalange of every of the four arms. The entire haptic memory check for each participant turned into videotaped; reminiscence considers was scored later using playback of the video recording (two technicians independently reviewed video playback of participants' classes and scored them for accuracy; consensus was completed in all cases). In order to check haptic memory, a participant inserted both of her arms palm-side up into the apertures at the base of the screen, permitting her hands to relaxation on the tabletop at the technician's aspect. The usage of the rounded-tip of a pitcher rod, the technician tapped the primary test sample on the right hand. The player withdrew her right hand (leaving the left hand in its resting position), and the usage of the proper index finger tapped the recalled sample on the road drawing above the

The participant changed her hand inside the aperture and the next sample become tapped at the left hand; with- drawing the left hand, the player used her left index finger to report on the road drawing above the left aperture. A practice pattern turned into administered for each player, and then the player turned into asked if she had any ques- tions before proceeding with the contact take a look at. The complete take a look at consisted of 24 consecutive styles; ordinary-numbered patterns have been administered to the proper hand even as even- numbered styles had been administered to the left hand. As a signal to the player, the pattern became introduced earlier than it was administered, e.g., "sample number one."

\subsection{Statistical Analyses}

A repeated-measures, small-n, inside-topics layout [29] turned into used for this take a look at to maximise the green use of research members (n 1/4 56). All statistical analyses have been performed with SPSS Version 10.0 [30]. All analyses were interpreted using a 1/4 0.05 so that all reports of significant results in this study are associated with a $\mathrm{p}<0.05$ (exact $\mathrm{p}$-values are reported for one-tail correlation tests).

Three hypotheses were posited in this study:

a. Hypothesis 1. Haptic-spatial memory performance differs among fingers and between hands. A 2 A 4 (Hand $\hat{A}$ Finger) repeated-measures analysis of variance was used to evaluate this hypothesis. In addition, a chi-square test was used to examine whether there were systematic finger substitution errors among participants, e.g., consistently indicating that the ring finger was tapped when actually the middle finger was tapped.

b. Hypothesis 2. A serial-position plot for haptic recall follows a classic curvilinear shape and demonstrates primacy and recency effects. For each participant, we tallied the number of taps correctly reported by serial position, with a total of 24 possible correct responses in each position. We used a $2 \hat{\mathrm{A}} 4$ (Hand $\hat{\mathrm{A}}$ Position) repeated-measures analysis of variance.

Tabel 1. Tap Recall by Finger and Hand

\begin{tabular}{ccc}
\hline Finger & Lefthand(M,SD) & RightHand(M,SD) \\
\hline Pinky & $10.75,1.78$ & $10.88,1.35$ \\
Ring & $10.25,1.86$ & $10.29,2.03$ \\
Middle & $10.70,1.35$ & $10.05,1.94$ \\
Index & $10.71,1.58$ & $10.55,1.65$ \\
\hline
\end{tabular}

Model to test this hypothesis. Specifically, we were interested whether information at the beginning or end will be recalled more efficiently and, therefore, we tested contrast codes for a quadratic model that captures the expected " $u$ " shape of recall as a function of ordinal position in a pattern. Hypothesis 3 , hapticspatial memory performance is correlated with short-and long-term visual-spatial memory performance. Using 55 participants (one of 56 participants in this study did not complete the Colorado Neuropyschology Test), we estimated cor-relation coefficients among three independent varia- bles: taps, VST scores (short- 
term visual-spatial), and MCT scores (long-term visual-spatial). For three independent variables, a sample size between 34 to 76 is adequate to detect medium to large effect sizes (f $2 \$ 0.15$ to 0.35 ; f 2 1/4 R 2 / ( 1 R 2)) with power (b) $1 / 40.80$ and a $1 / 40.05$ [31]; thus, thepresent study has reasonable statistical power. average wide variety of faucets recalled efficiently out of 96 faucets (24 styles $\hat{A}$ four finger taps) become M $1 / 484.04$ (SD $Æ$ 11.fifty six). Hereafter, those rankings for hap- tic reminiscence overall performance are called patterns and taps, respectively. The average faucets for the left or proper hand had been M 1/4 42.41 (SD 1/four 5. ninety seven) and M 1/four forty one.77 (SD 1/4 6.50), respectively; common faucets in step with finger are suggested in desk 1.

\subsection{Systematic Differences for Touch Recall}

There had been no sizeable differences for faucets between the left and right hand; but, there had been sizeable variations for faucets amongst arms, $\mathrm{F}$ (1, fifty five) 1 /four nine. $13, \mathrm{p}<0.05$. Fur- thermore, the Finger Â Hand interaction turned into giant, F $(3,55) 1 / 45.90, p<0.05$. In other words, there was no overall difference between hands for taps; however the difference in taps among fingers was different between hands. Specifi- cally, the tap recall performance for the middle finger of the left hand was notably better than the middle finger of the right hand (Table 1). In addition, errors were systematic among possible finger substitutions (Table 2); specifically, substitutions between ring and middle finger were more frequent than all other possible combinations (x 2 1/4 75.12, p < 0.05). Memory of touch of the pinky or index finger is recalled well than the memory of touch of the ring or middle finger.

Table 2. Systematic Errors for Tap Recall

\begin{tabular}{cccc}
\hline \multicolumn{3}{c}{ Substituted Finger } & \\
\hline Pinky & Ring & Middle & Index \\
Pinky & 53 & 34 & 44 \\
Ring & 51 & 94 & 54 \\
Middle & 36 & 94 & 53 \\
Index & 38 & 51 & 61 \\
\hline
\end{tabular}

Table 3. Number of Errors by Serial Position

\begin{tabular}{cccc}
\hline Position & $\mathrm{M}$ & $\mathrm{SD}$ & $\mathrm{n}$ \\
\hline First & 2.34 & 2.56 & 56 \\
Second & 3.02 & 3.27 & 56 \\
Third & 3.63 & 3.51 & 56 \\
Fourth & 2.88 & 3.05 & 56 \\
\hline
\end{tabular}

\subsection{Serial-Position Effect}

Among the four serial positions for each pattern, participants produced a greater variety of errors for tap keep in mind inside the $2 \mathrm{~d}$ and 0.33 positions compared to the first and fourth positions (table three). Specifically, tap recall proficiencies have been $90,87,85$, and 88 percentages for the primary thru fourth positions, respectively (Figure. 2), analogous to the serial role impact this is regularly stated for sequential phrase-remember tasks. A quadratic ANOVA version detected a massive serial-function impact $F(1,55) 1 / 4$ $22.14, \mathrm{p}<0.05$ for serial function, confirming a primacy and a recency effect for tap take into account performance. Using a one-tail test, tap is correlated significantly with VST scores (short-term visual-spatial memory; r 1/4 0.32, p 1/4 0.015) and MCT (long-term visual-spatial memory; r 1/4 0.26, p 1/4 0.061). Interestingly, the correlations between tap and either MCT or VST are greater than the correlation between VST and MCT and (r 1/4 0.22, p 1/4 0.112).

\section{CONCLUSION}

This exploratory have a look at changed into designed to assess haptic- spatial memory the use of simple and strange undertaking, patterns comprising 4 faucets throughout the centre phalanges. The gift examine affords sizable proof assisting three hypotheses: 1) Haptic-spatial reminiscence overall performance for this venture is depending on which finger is tapped and bear in mind mistakes are systematic; 2) the traditional phenomenon of a serial-role effect exists for the don't forget of those patterns; and three) there may be a sizeable and nice correlation among overall performance on this task and visual-spatial reminiscence. Two novel observations on this take a look at are that faucet take into account differs drastically with the aid of finger but now not through hand and the distribution of error in consider is depending on finger. The varyence with the aid of finger but now not via hand adds to the finding through 
that fingers and fingers separately method the identity of tactile stimuli.the character of the full-size errors dependency between the middle and ring hands may be due in element to ambiguity springing up from their physical proximity, their organizational proximity in the somatosensory cortex and the overlap of the innervation discipline for sensory input served in not unusual by using the medial nerve branch for these two hands. Amongst all arms, faucet do not forget overall performance was highest for the pinky, the only finger whose palmar surface comprises an innervation subject that is totally covered by theulnar nerve .some other viable supply of systematic blunders among the middle and ring arms is probably discovered in a capability position of acquainted motor pastime in recalling and reporting strange haptic styles. For examination ple, theme dial digits (center and ring fingers) percentage a enormously coordinated and acquainted motor pastime for greedy gadgets while the lateral digits (pinky and index hands), responding to outside perturbations exercise independent motor responses to counteract external torque accordingly, the cognitive system of reporting the reminiscence of an unfamiliar haptic-spatial pattern may interact a familiar anticipatory proprioceptive motor sign that is more likely to be harassed among medial digits in comparison to the lateral digits; the degree of this impact is probably stimulated by means of the technique of reporting, e.g., pointing, finger tapping, or verbalizing. The literature indicates that this systematic mistakes associated with an unfamiliar haptic-spatial assignment can be reduced with exercise. As example, using electromyography and transcranial magnetic stimulation, suggested that expert piano players observing video clips with piano-fingering errors produce a time-locked, somatotopic corticospinal signature corresponding mainly to the finger making the error, an effect that isn't always detected in non-pianist na€ 1ve contributors who obtained visible but now not motor training. With significant exercise, motor education changes the output sign despatched from the number one motor cortex to the corticospinal tract sooner or later altering the organisation of the corticomuscular pathway underlying an anticipatory proprioceptive haptic conduct e.g., pianofingering. With substantial sensori moto reduction, the brain develops a capacity to screen sensory input and anticipatory motor output which will mitigate error in acquainted haptic-spatial manipulations of the palms. Contributors on this take a look at could not see their palms whilst they had been tapped behind a display screen. however, faucet-take into account performance-indicative of express, short-term haptic spatial memory is correlated appreciably with performance on each short-time period (VST) and long-term (MCT) specific visible-spatial duties administered after the haptic-spatial test, i.e.,the haptic- and visible-spatial duties have been sequential and for that reason unbiased. among these 3 exams of reminiscence-faucet-bear in mind, VST, and MCT-the lengthy-time period visible-spatial memory check (MCT) is notably different because the participant is actively engaged for the duration of the encoding step (flipping cards the use of the mouse while viewing and comparing cards); therefore, the MCT check is a simultaneous visual-motor spatial challenge. In assessment, for the alternative two tests (tap take into account and VST), the player should pay interest in the course of encoding at the same time as some other agent (character or laptop) offers the tactile or visible sample and then reviews with a proprioceptive motor reaction (pointing with a finger at a schematic hand or manipulating an arrow on a laptop display with amouse). This similarity among the faucet bear in mind and VST checks may in component give an explanation for the more potent correlation that they share, compared with either's correlation with MCTorthecorrelation between VST and MCT.faucet-consider performance as measured in the gift look at is a easy and perhaps standard measure of spatial perfor-mance whose association with both visible-spatial degree is more than the correlation between the visual-spatial measures themselves. The correlation of medium impact length between faucet and visual-spatial memory as stated right here is consistent with an underlying cognitive gadget for processing spatial statistics. Although interesting, these outcome sought to be evaluated similarly in subsequent research designed to evaluate tapdon't forget overall performance in opposition to a battery for a sort of measures of visible and even auditoryspatial reminiscence. also worth of additional examine, faucet don't forget as measured on this take a look at is likely associated with overall performance for finger tapping, a easy proprioceptive assignment used extensively in neuroimaging research of motor pastime. Finger tapping is purported to make use of the soreferred to as visuo-spatial sketchpad of running memory that is critical for complex cognitive task, e.g., mathematical processing. The application of faucet remember as a robust measure for processing spatial information is implied by using the file that Taptalk seasoned-ficiency is correlated significantly with ACT ratings, a surrogate degree of intelligence.on this study, members mentioned haptic-spatial patterns through pointing to a figure of a corresponding hand suspended in the vertical plane (Figure. 1); this way of report become selected for 2 motives: 1) it is similar to the action of a "speaker" the usage of Taptalk, and 2) it gives a clean file of the player's bear in mind as captured through video. This technique was selected over clearly asking the participant to hold her hand desk bound at the same time as shifting fingers to record the order of the faucets-the latter venture is difficult, e.g., raising the hoop finger without simultaneously moving either the middle and pinky arms along with it. It's far mentioned that our approach of reporting disrupts the egocentric body wherein the sample turned into administered.subsequent research ought to study the role of report in regard to the outcomes of this take a look at. extra studies need to additionally contain patterns of 
more and more more tap period and numerous lengths of postpone between sensory input and reporting that allows you to estimate potential and period of haptic-spatial reminiscence. further-extra, next studies could simultaneously incorporate visual-spatial input to investigate reciprocal interactions between visible-haptic reference frames.even as the boom of haptic psychology has been observed by using psychophysical research and its ongoing improvement and refinement calls for extra research regarding the cognitive procedures associated with touch. A person may be thought of as a facts-processing device capable of interacting with and shaping its environment but this gadget has limits to its overall performance capability that hinge on cognitive strategies. Statistics-processing postulates may beused to describe underlying mechanisms of those cognitive procedures, which is specifically suitable when regular performance consequences are observed throughout a wide type of conditions; such is the case with the traditionalserial-function impact. yet plenty of what we know approximatelycognitive methods for this system pertains to the visible and auditory modalities, a bias in cognitive psychology that has persisted for many years .simple research concerning touch can increase without problems into several conventional subjects in cognitive psychology inclusive of interest and attention, memory period and capacity, vigilance and goal identification (e.g., signal detection idea), expertise representation, and language development based totally on touch, a notably unfa- miliar sensory modality for communication. For instance, its miles diagnosed in cognitive psychology thatactivities that start with controlled effort grow to be increasingly more automated in their execution with practice [2, p. 99]. Given the correlation among touch and vision for spatial reminiscence overall performance suggested right here and visuo-haptic interactions reported some place else, e.g., it is interesting to recall the relative contributions of touch and vision to the improvement of automaticity in everyday sports, along with texting, playing a piano or a online game, or the faraway operation of drones. In addition-more, basic cognitive research may be employed to evaluate the capacity for haptic operating memory and our ability to shift interest from one modality to another. That is in particular applicable for the development of haptic technology utilized to shift sensory load from thevisible and auditory modalities to the tactile modality particularly important for development in safety andoverall performance in stressful environments in which vision or listening to is hindered.

\section{REFERENCES}

1. G. Werner and B. L. Whitsel, "Functional organization of the somatosensory cortex," in Handbook of Sensory Physiology, vol. 2: Somatosensory System, A. Iggo, Ed. New York, NY, USA: Springer-Verlag, 1973.

2. J. R. Anderson, Cognitive Psychology and Its Implications. New York, NY, USA: Worth Publishers, 2005.

3. L. A. Jones, "Communication via the skin: The challenge of tactile displays," in Advanced Decision Architectures for the Warfighter: Foundations and Technology, P. McDermott and L. Allender, Eds., Adelphi, MD, USA: Army Res. Labc., 2008.

4. A. Moscatelli, A. Naceri, and M. O. Ernst, "Path integration in tactile perception of shapes," Behavioural Brain Res., vol. 274, pp. 355-364, 2014.

5. R. Langner, M. A. Sternkopf, T. S. Kellermann, C. Grefkes, F. Kurth, F. Schneider, K. Zilles, and S. B. Eickhoff, "Translating working memory into action: Behavioral and neural evidience for using motor representations in encoding visuo-spatial sequences," Human Brain Mapping, vol. 35, pp. 3465-3484, 2014. 